\title{
State-of-the-art review: stress T1 mapping-technical considerations, pitfalls and emerging clinical applications
}

\author{
Stefan K. Piechnik ${ }^{1} \cdot$ Stefan Neubauer $^{1} \cdot$ Vanessa M. Ferreira $^{1}$
}

Received: 13 March 2017 / Revised: 21 August 2017 / Accepted: 24 August 2017 / Published online: 15 September 2017

(C) The Author(s) 2017. This article is an open access publication

\begin{abstract}
In vivo mapping of the myocardial T1 relaxation time has recently attained wide clinical validation of its potential utility. In this review, we address the basic principles of the T1 mapping techniques, with particular attention to the emerging application of vasodilatory stress agents to interrogate the myocardial microvascular compartment, and differences between commonly used T1 mapping methods when applied in clinical practice.
\end{abstract}

Keywords Cardiovascular magnetic resonance - Vascular reactivity $\cdot$ Stress $\cdot$ Tissue characterization $\cdot$ T1 mapping

\section{Introduction: what is T1 mapping?}

T1 relaxation time, spin-lattice relaxation time, or simply $\mathrm{T} 1$, is the fundamental magnetic resonance property that describes the exponential recovery of the longitudinal component of magnetization $(\mathrm{Mz})$ back towards its thermal equilibrium. In vivo, the recovery of $\mathrm{Mz}$ is complex, but characterizing the underlying processes with a single $\mathrm{T} 1$ value has shown promise as a biomarker [1]. The measured $\mathrm{T} 1$ is determined by intrinsic tissue properties, and the extrinsic environment, including surrounding structure and milieu, as well as software and hardware used to measure T1. Modern sequences allow direct generation of spatially resolved $\mathrm{T} 1$ relaxation maps. T1 mapping of tissues allows immediate assessment of their T1 values on a voxel-by-voxel basis as

Stefan K. Piechnik

stefan.piechnik@cardiov.ox.ac.uk

1 Oxford Centre for Clinical Magnetic Resonance Research (OCMR), Division of Cardiovascular Medicine, Radcliffe Department of Medicine, University of Oxford, John Radcliffe Hospital, Oxford OX3 9DU, UK a method of direct quantitative tissue characterization. In general, each tissue type is expected to have a normal range of $\mathrm{T} 1$ values, deviation from which may indicate disease or a change in physiology.

\section{T1 mapping sequences}

Myocardial T1 values measured in vivo depend on the chosen method, and are influenced by technical factors, such as magnetic field strength and pulse sequence design, and physiologic factors, including heart rate, temperature, age, gender, and disease [1]. The general design of T1 mapping sequences includes delivery of a pre-pulse and acquisition of multiple T1-weighted images to allow fitting of these signals to an exponential recovery curve. Common T1 mapping sequences used for cardiac T1 mapping are inversion recovery techniques [2-5], saturation recovery techniques [6], and mixed hybrid approaches [7].

Current cardiac T1 mapping techniques evolved from the original Look-Locker spectroscopic method developed in 1970 [8], and provide a time-efficient approach for T1 mapping. As the living heart is a dynamic organ that contracts and relaxes, modification of the original scheme was needed to assure acquisition of sufficient information without sacrificing accuracy and clinical utility. The modified Look-Locker inversion recovery (MOLLI) was developed in 2004 [2] to address this issue by introducing intermittent image acquisition using electrocardiographic (ECG) gating to target a designated phase of the cardiac cycle, and then repeating the inversion experiments after a carefully optimized delay time to obtain adequate information to fit a single exponential T1 recovery curve (Fig. 1a). This sequence scheme significantly advanced $\mathrm{T} 1$ mapping for 


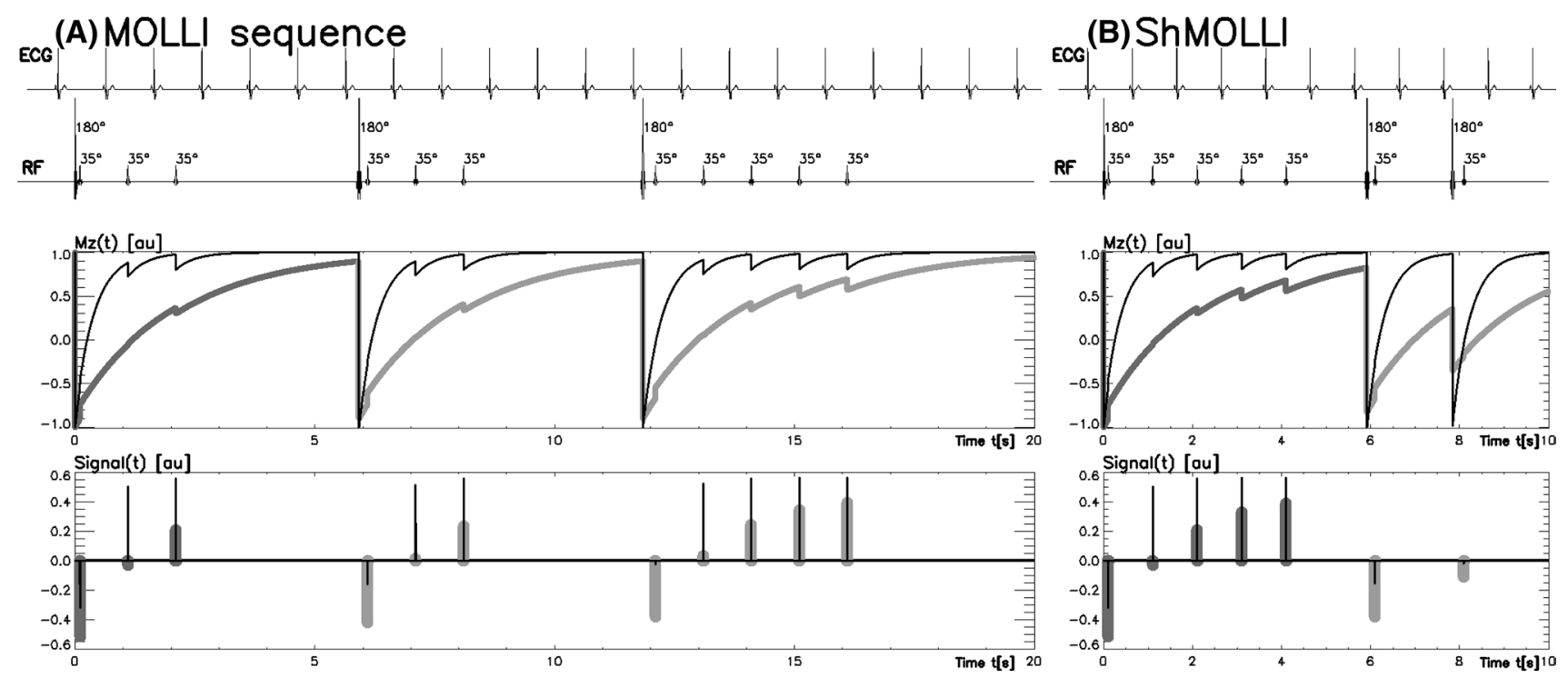

Fig. 1 ECG-gated pulse sequence schemes for simulation of a MOLLI and b ShMOLLI at a heart rate of $60 \mathrm{bpm}$. SSFP readouts are simplified to a single $35^{\circ}$ pulse each, presented at a constant delay time TD from each preceding $\mathrm{R}$ wave. The $180^{\circ}$ inversion pulses are shifted depending on the inversion recovery (IR) number to achieve the desired first TI of 100, 180 and $260 \mathrm{~ms}$ in the consecutive IR experiments. The plots below represent the evolution of longitudinal magnetisation (Mz) for short $\mathrm{T} 1$ (400 ms, thin lines) and long T1

cardiac applications, allowing acquisition of a cardiac T1 map within a manageable 17-heartbeat-long breathold.

The shortened modified Look-Locker inversion recovery (ShMOLLI 2010) [3] (Fig. 1b) further addressed several limitations of MOLLI-based cardiac T1 mapping towards practical clinical applications, and has been extensively validated clinically over the past 7 years [9-29]. In particular, advantages of ShMOLLI include:

1. Short breath-holds: it significantly shortened the breathhold time to 9 heartbeats (usually around $10 \mathrm{~s}$ ) per T1 map, rendering imaging time easier for sicker patients to cope with [3].

2. Heart rate independence: it eliminated heart rate dependency characteristic of other MOLLI-based techniques and variants, and is able to cope with tachyarrhythmias, such as rapid atrial fibrillation, frequent ectopic beats, and sinus tachycardia; this is particularly relevant for performing mapping during dynamic heart rate changes, such as for stress applications [3, 14, 27-29].

3. Flexibility: ShMOLLI is a one-for-all technique for a wide range of T1. In particular, it estimates long T1s without the progressive heart rate-dependent underestimation typical of MOLLI [3]. This is relevant for tissues such as blood (in the range of up to $2000 \mathrm{~ms}$ ) and fluids such as in pericardial effusions, cysts, and cerebrospinal fluid (in the range of 3000-4000 ms). This feature
(2000 ms, thick lines). Note that long epochs free of signal acquisitions minimise the impact of incomplete $\mathrm{Mz}$ recoveries in MOLLI so that all acquired samples can be pooled together for $\mathrm{T} 1$ reconstruction. In ShMOLLI, the validity of additional signal samples from the second and third IR epochs is determined by progressive nonlinear estimation. As originally published by BioMed Central in Piechnik [3]

is also important for assessing edematous tissues, and extracellular volume (ECV) where blood T1 is required. Other novel applications include characterization of masses (e.g. differentiating cysts from solid tumors) [30, 31], and splenic T1 to determine stress adequacy, which requires a $\mathrm{T} 1$ mapping sequence that can handle both long $\mathrm{T} 1$ values and in dynamic stress conditions [10, 11, 29, 32].

4. Practicality: ShMOLLI is able to simultaneously estimate short and long $\mathrm{T} 1$ pixels in the same image without requiring separate sequence sampling schemes for pre- and post-contrast $\mathrm{T} 1$ applications [33]; this makes it highly convenient in the practical workflow for ECV applications. It also allows post-contrast characterization of masses to determine gadolinium uptake, without misclassifying a cyst as a mass that appears to take up gadolinium contrast agents, which may, for example, suggest a vascular tumour on post-contrast T1 maps (Fig. 2).

There are other short MOLLI variants that have been developed also aimed at shortening imaging times [4, 33]. Currently, MOLLI-based sequences are the most commonly used and validated, although saturation-recovery single-shot acquisition (SASHA, SmarT1Map) sequences have attracted much attention due to acceptable short imaging times, nominal lack of heart rate dependency and excellent accuracy in 


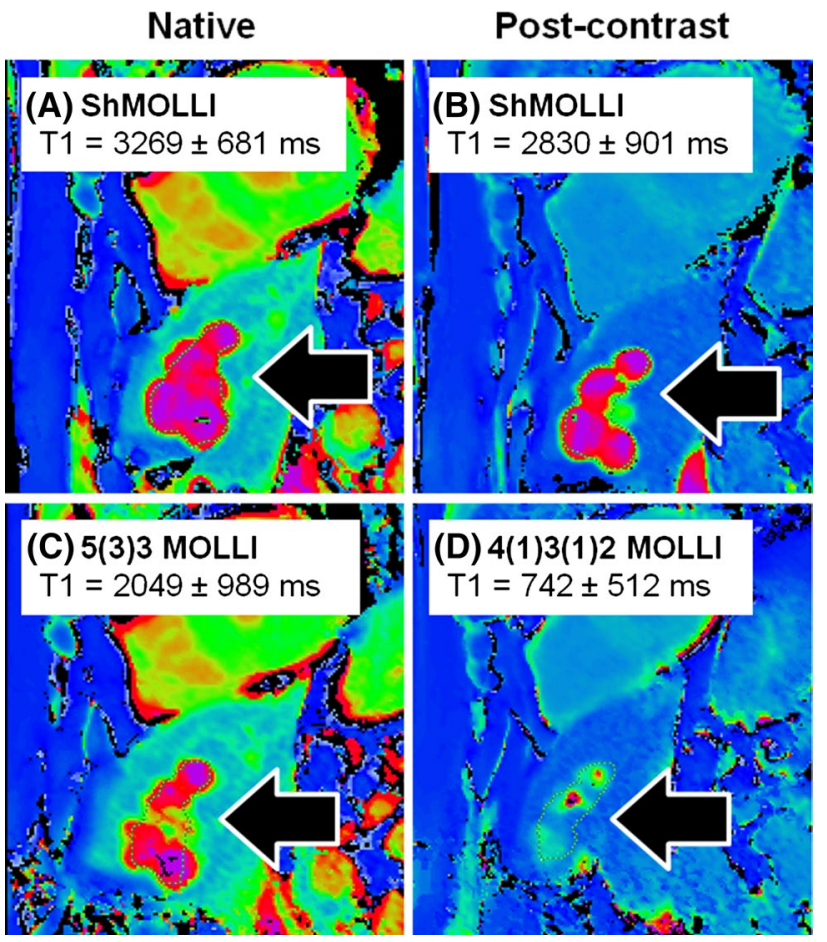

Fig. 2 Characterizing tissues with very long T1 values using different T1 mapping techniques. Shown are T1 maps from a patient with a past history of breast cancer. Liver cysts (black arrows) observed with ShMOLLI retains the characteristic very long T1 both pre- (a) and post-gadolinium-based contrast due to its consistent performance over a wide range of heart rates and T1 values. In c, the 5(3)3 MOLLI variant pre-contrast $\mathrm{T} 1$ map shows $\sim 30 \%$ lower $\mathrm{T} 1$ in the liver cysts, consistent with the back-loaded 11-heartbeart MOLLI 3(3)5 variant [4]. d Post-contrast T1 map using the 4(1)3(1)2 MOLLI variant dedicated for post-contrast applications suffers substantial underestimation of cystic $\mathrm{T} 1$ by $>70 \%$. Comparing $\mathbf{c}$ and $\mathbf{d}$, cystic lesions may appear to take up gadolinium-based contrast agents (GCBAs), which may suggest a tumour with communication to the vasculature, rather than what would be expected for a cyst. T1 is quoted for manual regions of interests drawn within the cysts. Colour tables are identical for all panels shown, as in Siemens ShMOLLI distributions for ease of comparisons

estimating myocardial T1 times shown in simulation and in phantoms [34, 35]. Hybrid approaches that combine saturation and inversion pulses are also available as emerging techniques for cardiac applications [7].

\section{What do T1 measurements bring to clinical practice?}

Myocardial T1 mapping methods can be used for native (or pre-contrast) T1 mapping, post-contrast T1 mapping, and ECV mapping (detailed review may be accessed elsewhere [36]). Briefly, native (pre-contrast) T1 reflects a composite signal from both the intracellular (predominantly myocytes) and extracellular spaces (which includes the interstitial and intravascular compartments). T1 predominantly detects free water, and increased free water content in tissue, such as edema or water collecting in expanded interstitial spaces. T1 does not directly detect collagen fibers, but predominantly the accumulation of water around fibrotic tissue which typically prolongs native $\mathrm{T} 1$ relaxation times and is responsible for the strong indirect links to areas of fibrosis reported in the literature. Processes that are known to lower T1 times include significant iron and fat content [26, 37, 38], as well as contrast agents, particularly gadolinium. Isolated, single time-point post-contrast T1 mapping is currently not preferred to estimating the ECV, due to strong dependencies on the timing and dose of contrast administered, and other confounding factors [1]. Instead, ECV may be quantified non-invasively using pre- and post-contrast $\mathrm{T} 1 \mathrm{maps}$ to obtain pre- and post-contrast myocardial and blood $\mathrm{T} 1$ values, adjusting for the hematocrit.

It is important to emphasize that $\mathrm{T} 1$ biomarkers are nonspecific and may deviate from their normal ranges due to a variety of causes. In particular, T1 and ECV may act as a surrogate for interstitial fibrosis only if other confounding factors of increased $\mathrm{T} 1$ or ECV-including edema, inflammation, amyloidosis that expand the interstitial space, and ischemia-have been excluded [1, 27, 28]. Current evidence demonstrates that native myocardial $\mathrm{T} 1$ values can be measured within a tight normal range, with clinically relevant sensitivity to changes in a wide range of cardiac diseases $[36,39,40]$. T1 maps can be displayed using color scales or threshold-based overlay masks to highlight tissue differences and aid visual interpretation [11, 13, 21, 31, 41, 42] (Fig. 3). Native T1 maps allow differentiation of an increasing range of tissue types without the need for gadolinium-based contrast agents (GBCA).

\section{Principle of gadolinium-free T1 mapping to assess the coronary vascular compartment}

Myocardial blood volume (MBV) constitutes $\sim 10 \%$ of the total myocardial volume at rest [43], and may increase twofold during coronary vasodilatory stress [44, 45]. In healthy individuals with normal myocardium and coronary arteries, there is significant coronary vasodilatory reserve, which can be interrogated by administration of adenosine vasodilatory stress [46]. Coronary vasodilation augments both coronary blood flow as well as intramyocardial blood volume [45]. Since native blood $\mathrm{T} 1$ is much longer than native myocardial $\mathrm{T} 1$, blood $\mathrm{T} 1$ is expected to increase the measured myocardial T1 through its partial volume effects [9]. This has been shown in normal volunteers who exhibit a $6 \%$ increase in myocardial $\mathrm{T} 1$ with narrow normal ranges during adenosine vasodilator stress, using the heart rate-independent 


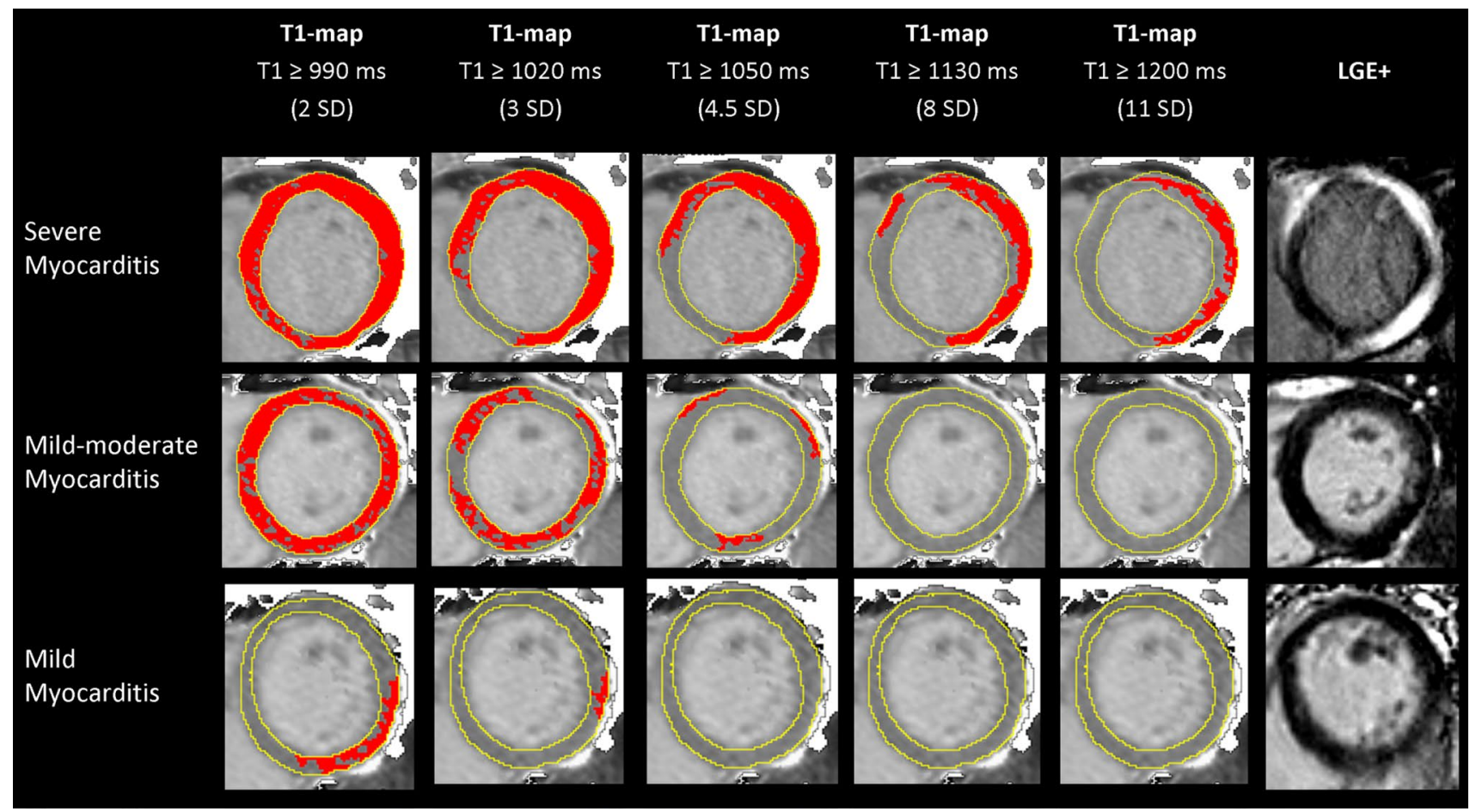

Fig. 3 T1 maps using incremental thresholds demonstrate the predominantly non-ischaemic pattern of injury across a spectrum of acute myocarditis. Red indicates areas of myocardium with a T1 value above the stated threshold of at least $40 \mathrm{~mm}^{2}$ in contigu-

ShMOLLI method $(6.2 \pm 0.5 \%$ at $1.5 \mathrm{~T} ; 6.3 \pm 1.1 \%$ at $3 \mathrm{~T})$ [28] (Fig. 4).

\section{Stress and rest $\mathrm{T} 1$ mapping in coronary artery disease (CAD)}

Stress T1 mapping has obvious potential applications in patients with CAD and ischemic heart disease [28, 47]. Liu et al. [28] demonstrated that, in normal myocardium, the resting $\mathrm{T} 1$ is normal, with a $6 \%$ rise during vasodilatory stress. In chronic infarcted myocardium, the resting $\mathrm{T} 1$ is typically significantly elevated compared to normal myocardium, with no change in T1 during stress (Fig. 4). In ischaemic myocardium subtended by a significant coronary stenosis, there is compensatory downstream coronary vasodilation even at rest; this is detectable as mildly elevated resting myocardial $\mathrm{T} 1$ values, but do not show further coronary vasodilatory response during stress, and, thus, no change in stress myocardial T1. Adenosine stress and rest T1 mapping may be used to distinguish normal, infarcted, and ischaemic myocardium, without the need for GCBA, due to their distinctive rest and stress T1 profiles [28] (Fig. 4). ous area. A T1 threshold of $990 \mathrm{~ms}$ was previously validated for the detection of acute myocardial oedema; other thresholds were selected for illustrative purposes. As originally published by Biomed Central in Ferreira et al. [13]

\section{Stress and rest $\mathrm{T} 1$ mapping in patients without obstructive CAD}

Adenosine stress and rest T1 mapping may also be used to assess coronary vasodilatory reserve in patients without obstructive CAD. For instance, in patients with type 2 diabetes without obstructive $\mathrm{CAD}$, early data have shown blunted stress $\mathrm{T} 1$ responses compared to controls, which may reflect microvascular dysfunction [48, 49], and is a subject of further investigation. In patients with severe aortic stenosis but no obstructive $\mathrm{CAD}$ on invasive angiography, the increased demands of the pressure-overloaded and hypertrophied myocardium are accompanied by increased resting coronary blood flow and vasodilation [50-52]. This is detectable as elevated resting myocardial $\mathrm{T} 1$, but achieving the same maximal adenosine stress $\mathrm{T} 1$ response when compared to normal controls [27]. This impaired stress $\mathrm{T} 1$ response normalizes 7 months after relief of the pressure overload with aortic valve replacement [27] (Fig. 5). This finding supports the notion that, in severe aortic stenosis, increased resting myocardial $\mathrm{T} 1$ may mainly reflect changes in the intravascular compartment, rather than solely from diffuse myocardial fibrosis in the interstitial compartment as previously believed, although these two processes likely co-exist in this disease model. Other investigators have explored stress T1 
(A)

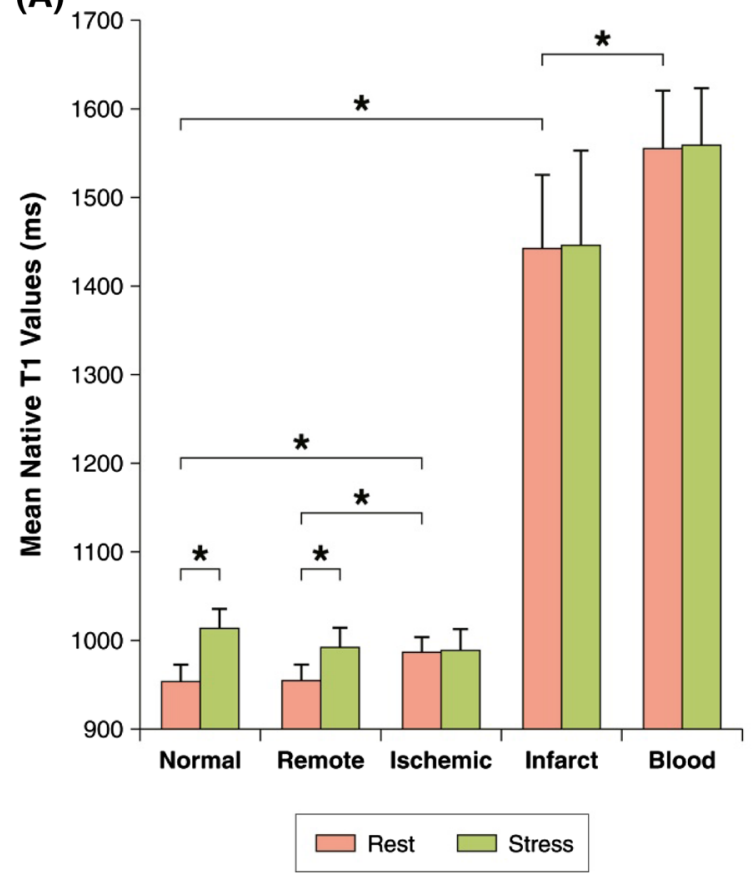

Fig. 4 Myocardial T1 at rest and during adenosine stress at $1.5 \mathrm{~T}$. a $\mathrm{T} 1$ values at rest in normal and remote tissue were similar and significantly lower than in ischemic regions. Infarct $\mathrm{T} 1$ was the highest of all myocardial tissue, but lower than the reference left ventricular blood pool of patients. During adenosine stress, normal and remote myocardial T1 increased significantly from baseline, while $\mathrm{T} 1$ in

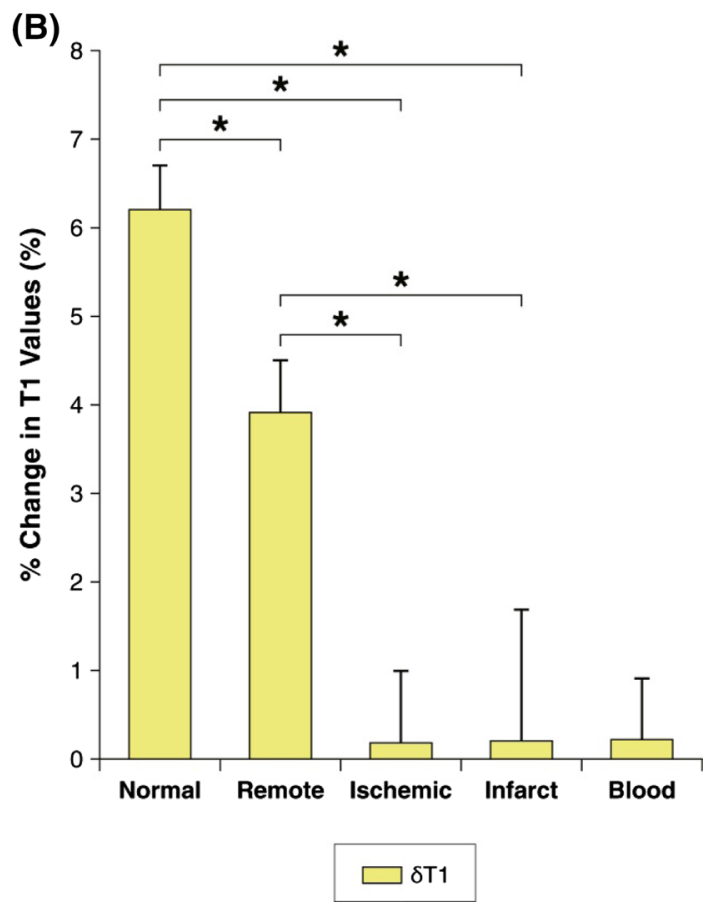

ischemic and infarcted regions remained relatively unchanged. b Relative T1 reactivity ( $\delta \mathrm{T} 1)$ in the patient's remote myocardium was significantly blunted compared to normal, and completely abolished in ischemic and infarcted regions. All data indicate mean \pm 1 SD. ${ }^{*} p<0.05$. As originally published by Elsevier in Liu [28]

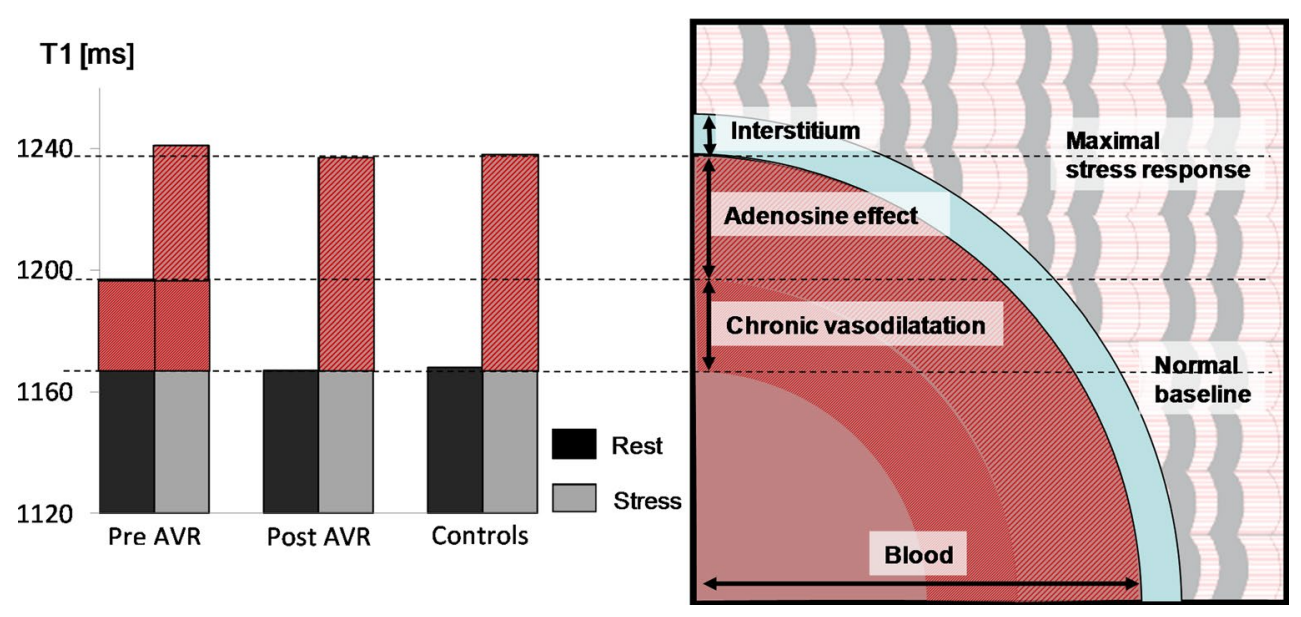

Fig. 5 Proposed myocardial water compartments in aortic stenosis. Proposed changes in myocardial water compartments at rest and stress in patients with aortic stenosis pre and post AVR, and controls $(l e f t)$. The $\mathrm{T} 1$ response to adenosine was mainly contributed to by

mapping as a surrogate marker for myocardial blood volume change in heart transplant recipients [48, 49]. We believe that stress T1 mapping holds promise for assessing coronary vascular responses instead of interstitial space expansion which may be negligible. Note that $\mathrm{T} 1$ and volumes from vascular cross-sections are for qualitative comparison only and not to scale. As originally published by BioMed Central in Mahmod et al. [27]

microvascular function and vasodilatory reserve in a number of cardiomyopathies as emerging clinical applications. 


\section{Splenic T1 mapping: a novel surrogate marker for adequate adenosine stress}

Stress adequacy is an integral component of the cardiac stress examination, which may impact on the diagnostic confidence, especially for ruling out significant obstructive CAD. Recently, stress T1 mapping of the spleen has been shown to be promising novel invention for assessing adenosine stress adequacy before stress perfusion clinical magnetic resonance (CMR) imaging [29] (Fig. 6). Whilst adenosine stress induces vasodilation in the coronary arteries, it simultaneously induces vasoconstriction in the spleen. This is manifest as the "splenic switch-off sign", which can be seen on nuclear stress perfusion [53] as well as CMR gadolinium-based perfusion imaging, and may serve as a marker for adenosine stress adequacy [54]. On CMR perfusion images, the spleen is typically visible in the field of view, and during peak adenosine stress, the spleen appears dark ("switch-off") compared to rest perfusion images when the spleen appears bright (as it takes up GBCA). The lack of "splenic switch-off" has been observed in more false negative perfusion CMR scans when compared to invasive coronary angiography in detecting significant CAD [54]. One limitation of the gadolinium-based "splenic switchoff" sign is that to visualize this interesting phenomenon, GBCA would have already been administered for first-pass perfusion imaging, and does not leave an opportunity to optimize the adenosine stress protocol on the fly. Splenic
T1 mapping, on the other hand, does not require GBCA, and splenic vasoconstriction associated with adenosine stress significantly decreases splenic $\mathrm{T} 1$ values, which can be conveniently detected on stress T1 maps typically without additional planning [29]. This provides a pre-emptive opportunity to increase and/or prolong adenosine administration to achieve adequate adenosine stress before acquiring stress images to increase diagnostic confidence. Splenic T1 mapping is undergoing further validation for this indication.

\section{Pitfalls of stress T1 mapping}

The impact of the chosen T1 mapping technique on rest and stress $\mathrm{T} 1$

It is widely recognized that $\mathrm{T} 1$ mapping techniques, even within a method family like MOLLI-based sequences, have different properties and diverging norms $[1,55]$. The impact of this issue is particularly apparent for stress $\mathrm{T} 1$ applications, as illustrated by two recent studies that used different MOLLI techniques to perform adenosine stress T1 mapping: Liu et al. [28] performed stress T1 mapping using ShMOLLI to study normal volunteers compared to patients with CAD, while Kuijpers et al. used MOLLI 5(3)3 stress T1 mapping to study patients who had normal findings on conventional CMR (served as controls) compared to CAD patients. Liu et al. observed a normal stress $\mathrm{T} 1$ response of
Fig. 6 Representative stress and rest splenic first-pass gadolinium perfusion and native T1 maps. Signal intensity (SI) curves represent splenic perfusion SI (y-axis, arbitrary units) over time ( $x$-axis, 50-60 s). The maximum and minimum $\mathrm{SI}_{\text {spleen }}$ are as indicated. Splenic regions of interests on perfusion images and $\mathrm{T} 1$ maps are outlined in red and black, respectively. Mean native $\mathrm{T} 1_{\text {spleen }}$ and stress changes $\left(\Delta \mathrm{T} 1_{\text {spleen }}\right)$ are as labelled. $3 \mathrm{~T}$ images were used for illustration (observed $\Delta \mathrm{T} 1$ spleen and $\Delta$ SI ${ }_{\text {spleen }}$ are field strength-independent). As originally published by BioMed Central in Liu et al. [29] (color figure online)

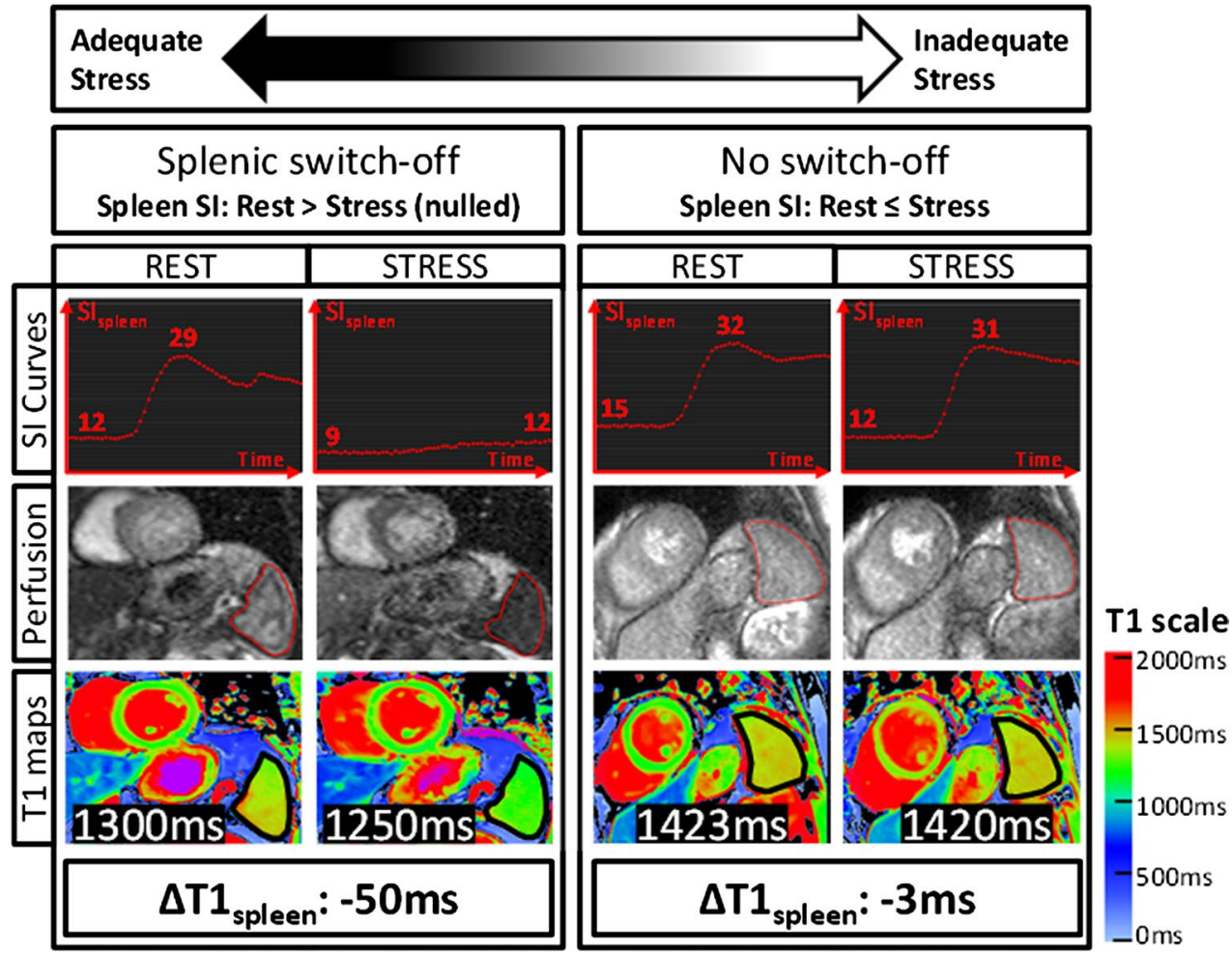


$6.2 \pm 0.5 \%$ at $1.5 \mathrm{~T}$ and $6.3 \pm 1.1 \%$ at $3 \mathrm{~T}$ [28], while Kuijpers et al. obtained a lower stress $\mathrm{T} 1$ response and larger standard deviations of $4.3 \pm 2.8 \%$ in controls at $1.5 \mathrm{~T}$ [47]. In CAD patients with remote myocardium, Liu et al. noted a blunted T1 response of $3.9 \pm 0.6 \%$, while Kuijpers et al. reported lower averages of $2.6 \pm 3.4 \%$. Similarly, another group of investigators reported that MOLLI 5(3)3 achieved a stress $\mathrm{T} 1$ response of 3.3\% (1.5 T) and 4.4\% (3 T) [47, 56]. Recently, stress $\mathrm{T} 1$ responses using regadenoson showed reactivity similar to those previously reported after adenosine administration [57].

It is encouraging that the stress $\mathrm{T} 1$ response can be elicited using more than one $\mathrm{T} 1$ mapping technique by independent groups of investigators and with different stress agents. At the same time, the fact that the ShMOLLI stress T1 response is larger by $>40 \%$ than using MOLLI 5(3)3 based on the published numbers above deserves attention and discussion. Conventionally, CMR methods that compare images before and after an intervention (such as administration of a stress agent or GBCA, as in perfusion imaging and ECV mapping) within the same subject in a single scan session may improve inter-individual and inter-center consistencies of the imaging biomarker. However, the same cannot be said for stress T1 mapping based on current limited evidence. In the two studies cited above [28, 46], the resting $\mathrm{T} 1\left(955 \pm 17 \mathrm{~ms}\right.$ in Liu et al. and $\mathrm{T} 1_{\text {rest }} 977 \pm 40 \mathrm{~ms}$ in Kuijpers et al.) amount to only a $2 \%$ difference between these two techniques. In contrast, the inter-methodological differences in stress $\mathrm{T} 1$ responses differ by $\sim 40 \%$ (approximately 6 vs. $4 \%$, respectively)—i.e., a 20 -fold worse agreement than for resting T1 values. Potential reasons for discrepancies in the stress $\mathrm{T} 1$ response between these two techniques may include factors such as selection of patients (with normal findings) as controls in the MOLLI study and control age differences, adenosine stress duration, adequacy, and maximal heart rate achieved; however, the technical differences between the ShMOLLI and MOLLI 5(3)3 T1 mapping techniques and their impact on the stress $\mathrm{T} 1$ response also require further consideration, as discussed below. Early standardization may be even more important for stress applications than for native resting $\mathrm{T} 1$.

\section{The impact of heart rate variation on stress $\mathrm{T} 1$ mapping}

ShMOLLI, with a sampling scheme of 5(1)1(1)1, is "frontloaded" by acquiring most samples upfront and is heart rate-independent due to its in-built conditional reconstruction algorithm $[3,28]$. This in contrast to earlier MOLLI techniques [58], which tend to be "back-loaded", with most samples acquired at the end of the sampling scheme, such as the classic MOLLI 3(3)3(3)5 design [2]. The MOLLI $5(3) 3$ variant aimed to reduce heart rate sensitivity also by front-loading [59] but does not ultimately eliminate it, as all data are used to fit to a single model, regardless of whether recovery epochs are adequately long or not (see Fig. 2). Given that stress T1 responses are relatively small, even a slight degree of residual heart rate sensitivity in the myocardial $\mathrm{T} 1$ range can impact on the observed stress $\mathrm{T} 1$ reactivity. Figure 7 illustrates the mechanism of stress T1 underestimation using 11-heart-beat 3(3)5 MOLLI due to heart rate dependency based on data published by an independent group of investigators [4]. The most recent MOLLI variant, using a sampling scheme of $5 \mathrm{~s}(3 \mathrm{~s}) 3 \mathrm{~s}$, has been proposed to further reduce heart rate sensitivity [60]. However, even within the relatively limited range of T1 $(0-1200 \mathrm{~ms})$ validated for this technique (further details in Fig. 9 in [60]), the $\mathrm{T} 1$ and heart rate dependence are still evident. It remains unclear what proportion of the significant $\mathrm{T} 1$ underestimation seen in wider $\mathrm{T} 1$ ranges beyond $1200 \mathrm{~ms}$, as reported by other studies for classic MOLLI [3, 4], may remain for MOLLI 5s(3s)3s.

SASHA T1 mapping is heart rate independent under optimal conditions, although there have been no reports of its application during dynamic stress thus far. It remains to be tested in practice by further comparisons whether the lower signal-to-noise ratio (SNR) known for this technique and whether imperfect saturation conditions may introduce confounds based on the known error dependencies (shown in Figs. 2, 3, 4 in the original paper [6]).

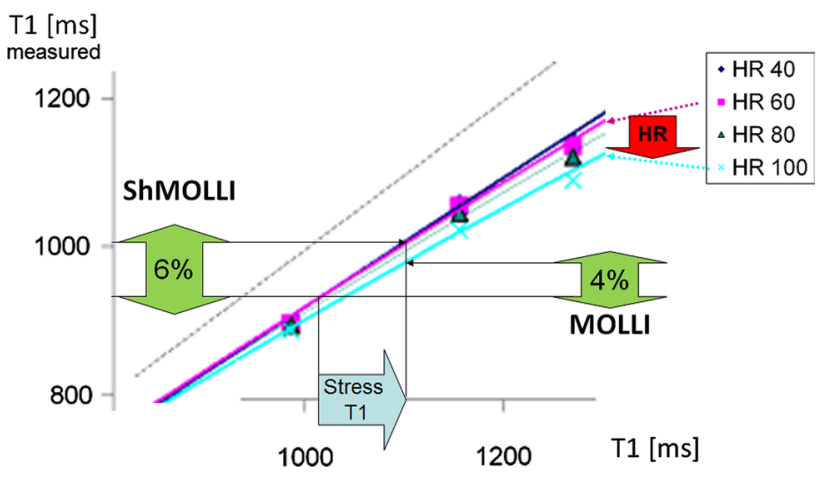

Fig. 7 Mechanism for the impact of heart rate sensitivity on the measured stress $\mathrm{T} 1$ responses using MOLLI variants. MOLLIs generally underestimate $\mathrm{T} 1$, hence all coloured lines are under the unity line (grey dotted). ShMOLLI has no heart rate (HR) dependence, and behaves like the HR 40 (dark blue) line across the HR range of 40-100 beats per minute. As a result, when myocardial T1 increases during vasodilatory stress (solid blue arrow, $x$-axis), this corresponds to just moving along a single linear relationship (dark blue HR 40), and preserves the relative size of the T1 response (6\%). The MOLLI 3(3)5 variant [4] illustrated here is HR dependent. Thus, when myocardial T1 increases during vasodilatory stress, the transition involves simultaneous switching between HR-dependent relationships (red arrow "HR"). This results in a lower $~ 4 \%$ stress $\mathrm{T} 1$ response using the MOLLI 3(3)5 variant. Adapted from Fig. 2 originally published by BioMed Central in Lee et al. [4] (color figure online) 


\section{Factors other than heart rate that impact on stress T1 mapping}

There are factors other than heart rate that may impact on the stress $\mathrm{T} 1$ response for a T1 mapping technique, which include $\mathrm{T} 1$ sensitivities to $\mathrm{T} 2$, magnetization transfer (MT) effects, and breath-hold duration and motion during stress conditions.

With regard to T2 sensitivities and MT effects, these properties that confer MOLLI-based techniques their recognized sensitivity to detecting disease [35] are likely to enhance their sensitivity to the stress T1 response elicited by ShMOLLI. Assuming that the underlying mechanism of the stress $\mathrm{T} 1$ response is mainly related to an increase in blood volume, the increased water content will directly affect MT and T2 to synergistically increase the measured T1. Further, the stress ShMOLLI-T1 response is likely to be enhanced by residual sensitivity to $\mathrm{T} 2$ elevations due to underlying BOLD (blood oxygenation level dependent) effects. The surplus BOLD response is characteristic of normal vascular reactivity [61-63] and will also accentuate the contrast to pathological changes. Conversely, while stress T1 mapping has not been reported using saturation-recovery techniques, the lack of MT and T2 dependencies will likely diminish the stress $\mathrm{T} 1$ response, especially given the higher variability (noise) typically seen in T1 estimation using saturationrecovery methods $[6,35,64]$. Recently improved inversion pulses in recent MOLLI variants target the accuracy of T1 by reducing T2 and MT sensitivities, which may be paradoxically detrimental to their sensitivity to detect stress $\mathrm{T} 1$ responses [35].

For the more recent MOLLI variants that use sampling schemes measured in seconds (rather than in heartbeats) [60], at increased heart rates, there will be more image acquisitions per inversion recovery experiment, and more energy deposited into bound proton pools. For example, MOLLI $5 \mathrm{~s}(3 \mathrm{~s}) 3 \mathrm{~s}$ would deploy as MOLLI 5(3)3 at a heart rate of 60 beats per minute, but at 120 beats per minute, MOLLI 10(6)6 would be deployed, with twice as many readouts. Thus, MT sensitivity between the actual variants deployed at rest and during stress is likely to differ significantly. What happens exactly is largely academic, as its clinical application is likely to be more impacted by the breath-hold requirements. Human subjects undergoing dynamic stress using the $5 \mathrm{~s}(3 \mathrm{~s}) 3 \mathrm{~s}$ sampling scheme would need to hold their breath typically for 12-14 s, longer than classic MOLLI under the same stress conditions. Kuijpers et al. [47] had reported substantial motion artefacts using MOLLI 5(3)3 for stress T1 mapping, and these are likely to worsen using MOLLI $5 \mathrm{~s}(3 \mathrm{~s}) 3 \mathrm{~s}$ due to longer breath-hold requirements. Recent studies agreed that motion remains a substantial concern for MOLLI acquisitions for stress applications, which could not be overcome by inline MOCO $[47,56]$.
It would be difficult to explain the observed dependencies quantitatively by simple partial-volume relaxivity summation. We draw attention to the complex spectrum of blood $\mathrm{T} 1, \mathrm{~T} 2$, and volumetric reactivity between various vascular compartments in the brain (details in Figs. 11-14 in [61], supplemental material). Briefly, these demonstrate very significant differences in baseline values and stress reactivity of $\mathrm{T} 1$ and $\mathrm{T} 2$ of blood in various vascular compartments in the brain, with a disparately small blood volume attributed to the vaso-reactive arterial component when compared to the capillary and venous bed. These effects in the brain have been studied in response to $\mathrm{CO}_{2}$ administration, but not with adenosine or the dynamically changing tissue stress that occurs with each heart beat. These factors are important, as the dynamics of compartmental volume redistribution depend on time scales and the types of stimuli in the brain [65]. Given the challenges to gather similar data for the heart, ultimately, the diagnostic performance of a method to study the heart during stress conditions will boil down to clinical evidence and independent head-to-head comparisons in clinical practice [64]. Computer simulations and phantom experiments, while helpful as initial guides to assess a new method, may not fully replicate or account for factors encountered in the in vivo environment [64], especially in a complex and dynamic organ like the human heart.

\section{Future directions and implications}

Stress and rest T1 mapping is a novel technique with potential to assess ischaemia, coronary vasodilatory reserve, and the health of the micro-coronary circulation, without the need for GCBA. T1 mapping is a nascent field, and the exact biological mechanisms of native and stress T1 signals in various conditions have not been fully elucidated. The effects of other modalities of stress, including exercise and pharmacological agents, as well as other modulators of vascular reactivity on $\mathrm{T} 1$ may be explored to fully determine its clinical applicability. Stress T1 mapping is an active area of scientific development, including validation against quantitative perfusion measures, invasive coronary measurements and diagnostic performance in a variety of cardiac conditions [55, 66-70]. Over time, collective evidence will allow better understanding of the mechanisms for the observed changes for this emerging technique and its clinical utility in a wider patient population, including those with contraindications to GBCA.

Acknowledgements The authors acknowledge support from the British Heart Foundation Centre of Research Excellence (Oxford, UK). The authors are supported by the NIHR Biomedical Research Centre, Oxford. 


\section{Compliance with ethical standards}

Conflict of interest Professor Stefan K. Piechnik has patent authorship rights for US patent 9285446 B2. Systems and methods for shortened Look-Locker inversion recovery (Sh-MOLLI) cardiac gated mapping of T1. Granted March 15, 2016. The patent is managed by Oxford University. All rights transferred exclusively to Siemens Medical. To date, SKP received $£ 4396.92$ for this IP, before tax and National Insurance deductions.

Open Access This article is distributed under the terms of the Creative Commons Attribution 4.0 International License (http://creativecommons.org/licenses/by/4.0/), which permits unrestricted use, distribution, and reproduction in any medium, provided you give appropriate credit to the original author(s) and the source, provide a link to the Creative Commons license, and indicate if changes were made.

\section{References}

1. Moon J, Messroghli D, Kellman P, Piechnik S, Robson M, Ugander M, Gatehouse P, Arai A, Friedrich M, Neubauer S, SchulzMenger J, Schelbert E (2013) Myocardial T1 mapping and extracellular volume quantification: a Society for Cardiovascular Magnetic Resonance (SCMR) and CMR Working Group of the European Society of Cardiology consensus statement. J Cardiovasc Magn Reson 15(1):92

2. Messroghli DR, Radjenovic A, Kozerke S, Higgins DM, Sivananthan MU, Ridgway JP (2004) Modified look-locker inversion recovery (MOLLI) for high-resolution T1 mapping of the heart. Magn Reson Med 52(1):141-146

3. Piechnik SK, Ferreira VM, Dall'Armellina E, Cochlin LE, Greiser A, Neubauer S, Robson MD (2010) Shortened Modified LookLocker Inversion recovery (ShMOLLI) for clinical myocardial T1-mapping at 1.5 and $3 \mathrm{~T}$ within a 9 heartbeat breathhold. $\mathrm{J}$ Cardiovasc Magn Reson 12:69

4. Lee J, Liu S, Nacif M, Ugander M, Han J, Kawel N, Sibley C, Kellman P, Arai A, Bluemke D (2011) Myocardial T1 and extracellular volume fraction mapping at 3 tesla. J Cardiovasc Magn Reson 13(1):75

5. Weingärtner S, Roujol S, Akçakaya M, Basha TA, Nezafat R (2015) Free-breathing multislice native myocardial T1 mapping using the slice-interleaved T1 (STONE) sequence. Magn Reson Med 74(1):115-124

6. Chow K, Flewitt JA, Green JD, Pagano JJ, Friedrich MG, Thompson RB (2014) Saturation recovery single-shot acquisition (SASHA) for myocardial T(1) mapping. Magn Reson Med 71(6):2082-2095

7. Weingärtner $S$, Akçakaya $M$, Basha $T$, Kissinger KV, Goddu B, Berg S, Manning WJ, Nezafat R (2014) Combined saturation/ inversion recovery sequences for improved evaluation of scar and diffuse fibrosis in patients with arrhythmia or heart rate variability. Magn Reson Med 71(3):1024-1034

8. Look DC, Locker DR (1970) Time saving in measurement of NMR and EPR relaxation times. Rev Sci Instrum 41(2):250-251

9. Piechnik SK, Ferreira VM, Lewandowski AJ, Ntusi NAB, Banerjee R, Holloway C, Hofman MBM, Sado DM, Maestrini V, White SK, Lazdam M, Karamitsos T, Moon JC, Neubauer S, Leeson P, Robson MD (2013) Normal variation of magnetic resonance $\mathrm{T} 1$ relaxation times in the human population at $1.5 \mathrm{~T}$ using ShMOLLI. J Cardiovasc Magn Reson 15:13

10. Dall'Armellina E, Piechnik SK, Ferreira VM, Si QL, Robson MD, Francis JM, Cuculi F, Kharbanda RK, Banning AP, Choudhury RP, Karamitsos TD, Neubauer S (2012) Cardiovascular magnetic resonance by non contrast T1-mapping allows assessment of severity of injury in acute myocardial infarction. J Cardiovasc Magn Reson 14:15

11. Ferreira V, Piechnik S, Dall'Armellina E, Karamitsos T, Francis J, Choudhury R, Friedrich M, Robson M, Neubauer S (2012) Noncontrast T1-mapping detects acute myocardial edema with high diagnostic accuracy: a comparison to T2-weighted cardiovascular magnetic resonance. J Cardiovasc Magn Reson 14(1):42

12. Ferreira VM, Piechnik SK, Dall' armellina E, Karamitsos TD, Francis JM, Ntusi N, Holloway C, Choudhury RP, Kardos A, Robson MD, Friedrich MG, Neubauer S (2013) T1 mapping for the diagnosis of acute myocarditis using CMR: comparison to T2-weighted and late gadolinium enhanced imaging. JACC Cardiovasc Imaging 6(10):1048-1058

13. Ferreira V, Piechnik S, Dall'Armellina E, Karamitsos T, Francis J, Ntusi N, Holloway C, Choudhury R, Kardos A, Robson M, Friedrich M, Neubauer S (2014) Native T1-mapping detects the location, extent and patterns of acute myocarditis without the need for gadolinium contrast agents. J Cardiovasc Magn Reson 16(1):36

14. Ferreira VM, Wijesurendra RS, Liu A, Greiser A, Casadei B, Robson MD, Neubauer S, Piechnik SK (2015) Systolic ShMOLLI myocardial T1-mapping for improved robustness to partial-volume effects and applications in tachyarrhythmias. J Cardiovasc Magn Reson 17:77

15. Dass S, Suttie JJ, Piechnik SK, Ferreira VM, Holloway CJ, Banerjee R, Mahmod M, Cochlin L, Karamitsos TD, Robson MD, Watkins H, Neubauer S (2012) Myocardial tissue characterization using magnetic resonance noncontrast $\mathrm{t} 1$ mapping in hypertrophic and dilated cardiomyopathy. Circ Cardiovasc Imaging 5(6):726-733

16. Bull S, White SK, Piechnik SK, Flett AS, Ferreira VM, Loudon M, Myerson SG (2013) Human non-contrast T1 values and correlation with histology in diffuse fibrosis. Heart 99(13):932-937

17. Karamitsos TD, Piechnik SK, Banypersad S, Fontana M, Ntusi N, Ferreira VM, Whelan C, Myerson SG, Robson MD, Hawkins $P$, Neubauer S (2012) Non-contrast T1 mapping for the diagnosis of cardiac amyloidosis. JACC Cardiovasc Imaging. doi:10.1016/j. jcmg.2012.11.013 (Published online before print)

18. Ntusi N, Piechnik S, Francis J, Ferreira V, Rai A, Matthews P, Robson M, Moon J, Wordsworth P, Neubauer S, Karamitsos T (2014) Subclinical myocardial inflammation and diffuse fibrosis are common in systemic sclerosis-a clinical study using myocardial T1-mapping and extracellular volume quantification. J Cardiovasc Magn Reson 16(1):21

19. Ntusi NA, Piechnik SK, Francis JM, Ferreira VM, Matthews PM, Robson MD, Wordsworth PB, Neubauer S, Karamitsos TD (2015) Diffuse myocardial fibrosis and inflammation in rheumatoid arthritis: insights from CMR T1 mapping. JACC Cardiovasc Imaging 8(5):526-536

20. Ntusi N, O'Dwyer E, Dorrell L, Wainwright E, Piechnik S, Clutton G, Hancock G, Ferreira V, Cox P, Badri M, Karamitsos T, Emmanuel S, Clarke K, Neubauer S, Holloway C (2016) HIV1-related cardiovascular disease is associated with chronic inflammation, frequent pericardial effusions, and probable myocardial edema. Circ Cardiovasc Imaging 9(3):e004430

21. Ferreira VM, Marcelino M, Piechnik SK, Marini C, Karamitsos TD, Ntusi NA, Francis JM, Robson MD, Arnold JR, Mihai R, Thomas JD, Herincs M, Hassan-Smith ZK, Greiser A, Arlt W, Korbonits M, Karavitaki N, Grossman AB, Wass JA, Neubauer S (2016) Pheochromocytoma is characterized by catecholaminemediated myocarditis, focal and diffuse myocardial fibrosis, and myocardial dysfunction. J Am Coll Cardiol 67(20):2364-2374

22. Fontana M, White S, Banypersad S, Sado D, Maestrini V, Flett A, Piechnik S, Neubauer S, Roberts N, Moon J (2012) Comparison of T1 mapping techniques for ECV quantification. Histological 
validation and reproducibility of ShMOLLI versus multibreathhold T1 quantification equilibrium contrast CMR. J Cardiovasc Magn Reson 14(1):88

23. Fontana M, Banypersad SM, Treibel TA, Maestrini V, Sado DM, White SK, Pica S, Castelletti S, Piechnik SK, Robson MD, Gilbertson JA, Rowczenio D, Hutt DF, Lachmann HJ, Wechalekar AD, Whelan CJ, Gillmore JD, Hawkins PN, Moon JC (2014) Native T1 mapping in transthyretin amyloidosis. JACC Cardiovasc Imaging 7(2):157-165

24. Banypersad SM, Fontana M, Maestrini V, Sado DM, Captur G, Petrie A, Piechnik SK, Whelan CJ, Herrey AS, Gillmore JD, Lachmann HJ, Wechalekar AD, Hawkins PN, Moon JC (2015) T1 mapping and survival in systemic light-chain amyloidosis. Eur Heart J 36(4):244-251

25. Sado DM, White SK, Piechnik SK, Banypersad SM, Treibel T, Captur G, Fontana M, Maestrini V, Flett AS, Robson MD, Lachmann RH, Murphy E, Mehta A, Hughes D, Neubauer S, Elliott PM, Moon JC (2013) Identification and assessment of andersonfabry disease by cardiovascular magnetic resonance noncontrast myocardial T1 mapping. Circ Cardiovasc Imaging 6(3):392-398

26. Sado DM, Maestrini V, Piechnik SK, Banypersad SM, White SK, Flett AS, Robson MD, Neubauer S, Ariti C, Arai A, Kellman P, Yamamura J, Schoennagel BP, Shah F, Davis B, Trompeter S, Walker M, Porter J, Moon JC (2015) Noncontrast myocardial T1 mapping using cardiovascular magnetic resonance for iron overload. J Magn Reson Imaging 41(6):1505-1511

27. Mahmod M, Piechnik SK, Levelt E, Ferreira VM, Francis JM, Lewis A, Pal N, Dass S, Ashrafian H, Neubauer S, Karamitsos TD (2014) Adenosine stress native T1 mapping in severe aortic stenosis: evidence for a role of the intravascular compartment on myocardial T1 values. J Cardiovasc Magn Reson 16(1):1-8

28. Liu A, Wijesurendra RS, Francis JM, Robson MD, Neubauer S, Piechnik SK, Ferreira VM (2016) Adenosine stress and rest T1 mapping can differentiate between ischemic, infarcted, remote, and normal myocardium without the need for gadolinium contrast agents. JACC Cardiovasc Imaging 9(1):27-36

29. Liu A, Wijesurendra RS, Ariga R, Mahmod M, Levelt E, Greiser A, Petrou M, Krasopoulos G, Forfar JC, Kharbanda RK, Channon KM, Neubauer S, Piechnik SK, Ferreira VM (2017) Splenic T1-mapping: a novel quantitative method for assessing adenosine stress adequacy for cardiovascular magnetic resonance. J Cardiovasc Magn Reson 19(1):1

30. Saba SG, Bandettini PW, Shanbhag SM, Spottiswoode BS, Kellman P, Arai AE (2015) Characterization of cardiac masses with T1 mapping. J Cardiovasc Magn Reson 17(1):Q32

31. Ferreira VM, Holloway CJ, Piechnik SK, Karamitsos TD, Neubauer S (2013) Is it really fat? Ask a T1-map. Eur Heart J Cardiovasc Imaging. doi:10.1093/ehjci/jet095

32. Ferreira VM, Piechnik SK, Dall'Armellina E, Karamitsos TD, Francis JM, Ntusi N, Holloway C, Choudhury RP, Kardos A, Robson MD, Friedrich MG, Neubauer S (2013) T1 mapping for the diagnosis of acute myocarditis using CMR: comparison to T2-weighted and late gadolinium enhanced imaging. JACC Cardiovasc Imaging 6(10):1048-1058

33. Kellman P, Arai AE, Xue H (2013) T1 and extracellular volume mapping in the heart: estimation of error maps and the influence of noise on precision. J Cardiovasc Magn Reson 15:56

34. Chow K, Flewitt JA, Green JD, Pagano JJ, Friedrich MG, Thompson RB (2013) Saturation recovery single-shot acquisition (SASHA) for myocardial T1 mapping. Magn Reson Med 71(6):2082-2095

35. Robson MD, Piechnik SK, Tunnicliffe EM, Neubauer S (2013) T1 measurements in the human myocardium: the effects of magnetization transfer on the SASHA and MOLLI sequences. Magn Reson Med. doi:10.1002/mrm.24867 (Epub ahead of print)
36. Ferreira VM, Piechnik SK, Robson MD, Neubauer S, Karamitsos TD (2014) Myocardial tissue characterization by magnetic resonance imaging: novel applications of $\mathrm{T} 1$ and $\mathrm{T} 2$ mapping. $\mathrm{J}$ Thorac Imaging 29(3):147-154

37. Sado DM, White SK, Piechnik SK, Banypersad SM, Treibel T, Captur G, Fontana M, Maestrini V, Flett AS, Robson MD, Lachmann RH, Murphy E, Mehta A, Hughes D, Neubauer S, Elliott PM, Moon JC (2013) Identification and assessment of AndersonFabry disease by cardiovascular magnetic resonance noncontrast myocardial T1 mapping. Circ Cardiovasc Imaging 6(3):392-398

38. Feng Y, He T, Carpenter J-P, Jabbour A, Alam MH, Gatehouse PD, Greiser A, Messroghli D, Firmin DN, Pennell DJ (2013) In vivo comparison of myocardial $\mathrm{T} 1$ with $\mathrm{T} 2$ and $\mathrm{T} 2 *$ in thalassaemia major. J Magn Reson Imaging 38(3):588-593

39. Piechnik SK, Ferreira VM, Lewandowski AJ, Ntusi NA, Banerjee R, Holloway C, Hofman MB, Sado DM, Maestrini V, White SK, Lazdam M, Karamitsos T, Moon JC, Neubauer S, Leeson P, Robson MD (2013) Normal variation of magnetic resonance T1 relaxation times in the human population at $1.5 \mathrm{~T}$ using ShMOLLI. $\mathrm{J}$ Cardiovasc Magn Reson 15:13

40. Dabir D, Child N, Kalra A, Rogers T, Gebker R, Jabbour A, Plein S, Yu C-Y, Otton J, Kidambi A, McDiarmid A, Broadbent D, Higgins DM, Schnackenburg B, Foote L, Cummins C, Nagel E, Puntmann VO (2014) Reference values for healthy human myocardium using a T1 mapping methodology: results from the International T1 Multicenter cardiovascular magnetic resonance study. J Cardiovasc Magn Reson 16(1):1-12

41. Ferreira VM, Piechnik SK, Firoozan S, Karamitsos TD, Neubauer S (2014) Acute chest pain and massive left ventricular hypertrophy in a 38 year-old man. Heart 100(4):347

42. Kellman P, Wilson J, Xue H, Bandettini W, Shanbhag S, Druey K, Ugander M, Arai A (2012) Extracellular volume fraction mapping in the myocardium, part 2: initial clinical experience. J Cardiovasc Magn Reson 14(1):64

43. Judd RM, Levy BI (1991) Effects of barium-induced cardiac contraction on large- and small-vessel intramyocardial blood volume. Circ Res 68(1):217-225

44. Crystal GJ, Downey HF, Bashour FA (1981) Small vessel and total coronary blood volume during intracoronary adenosine. Am J Physiol 241(2):H194-H201

45. McCommis KS, Zhang H, Goldstein TA, Misselwitz B, Abendschein DR, Gropler RJ, Zheng J (2009) Myocardial blood volume is associated with myocardial oxygen consumption: an experimental study with CMR in a canine model. JACC Cardiovasc Imaging 2(11):1313-1320

46. Wilson RF, Wyche K, Christensen BV, Zimmer S, Laxson DD (1990) Effects of adenosine on human coronary arterial circulation. Circulation 82(5):1595-1606

47. Kuijpers D, Prakken NH, Vliegenthart R, van Dijkman PRM, van der Harst P, Oudkerk M (2016) Caffeine intake inverts the effect of adenosine on myocardial perfusion during stress as measured by T1 mapping. Int J Cardiovasc Imaging 32(10):1545-1553

48. Levelt E, Piechnik SK, Mahmod M, Ferreira VM, Ariga R, Francis JM, Liu A, Sellwood J, Wijesurendra RS, Robson MD, Clarke K, Neubauer S, Karamitsos TD (2015) Adenosine stress native T1 mapping detects microvascular disease in diabetic cardiomyopathy, without the need for gadolinium-based contrast. J Cardiovasc Magn Reson 17(Suppl 1):Q55

49. Nayak K, Do HP, Yoon AJ, Fong M, Grazette L, Farhood S, Barr ML (2017) Vasodilator response in heart transplant recipients using T1-based myocardial blood volume mapping. In: Proceedings from the 20th annual SCMR scientific sessions, p 669

50. Rajappan K, Rimoldi OE, Dutka DP, Ariff B, Pennell DJ, Sheridan DJ, Camici PG (2002) Mechanisms of coronary microcirculatory dysfunction in patients with aortic stenosis and angiographically normal coronary arteries. Circulation 105(4):470-476 
51. Marcus ML, Doty DB, Hiratzka LF, Wright CB, Eastham CL (1982) Decreased coronary reserve: a mechanism for angina pectoris in patients with aortic stenosis and normal coronary arteries. N Engl J Med 307(22):1362-1366

52. Marcus ML, Koyanagi S, Harrison DG, Doty DB, Hiratzka LF, Eastham CL (1983) Abnormalities in the coronary circulation that occur as a consequence of cardiac hypertrophy. Am J Med $75(3 a): 62-66$

53. Gates GF, Ames AW (1986) Splenic "disappearance" during gated exercise nuclear angiocardiography. Clin Nucl Med 11(10):683-687

54. Manisty C, Ripley DP, Herrey AS, Captur G, Wong TC, Petersen SE, Plein S, Peebles C, Schelbert EB, Greenwood JP, Moon JC (2015) Splenic switch-off: a tool to assess stress adequacy in adenosine perfusion cardiac MR imaging. Radiology 276(3):732-740

55. Puntmann VO, Peker E, Chandrashekhar Y, Nagel E (2016) T1 mapping in characterizing myocardial disease. Compr Rev 119(2):277-299

56. Puntmann VO, Peker E, Winau L, Heinke R, Schnoes K, Wichmann JL, Vogl T, Zeiher AM, Greiser A, Nagel E (2017) T1 mapping at rest and adenosine stress-comparison of T1 mapping sequences for feasibility and effect size. In: Proceedings from the 20th SCMR anual scientific sessions, $p 241$

57. van Dijk R, Kuijpers D, Kaandorp TAM, van Dijkman PRM, Vliegenthart R, van der Harst P, Oudkerk M (2017) Effects of caffeine intake prior to stress cardiac magnetic resonance perfusion imaging on regadenoson- versus adenosine-induced hyperemia as measured by T1 mapping. Int J Cardiovasc Imaging. doi:10.1007/ s10554-017-1157-4

58. Liu S, Huang J, Ugander M, Sibley C, Oki A, Han J, Greiser A, Messroghli DR, Kellman P, Arai AE, Bluemke DA (2011) Phantom validation of 17 and 11 heartbeat MOLLI T1 mapping sequence at 3 T. J Cardiovasc Magn Reson 13(Suppl 1):P10

59. Kellman P, Wilson JR, Xue H, Ugander M, Arai AE (2012) Extracellular volume fraction mapping in the myocardium, part
1: evaluation of an automated method. J Cardiovasc Magn Reson 14(1):63

60. Kellman P, Hansen MS (2014) T1-mapping in the heart: accuracy and precision. J Cardiovasc Magn Reson 16(1):1-20

61. Piechnik SK, Chiarelli PA, Jezzard P (2008) Modelling vascular reactivity to investigate the basis of the relationship between cerebral blood volume and flow under $\mathrm{CO}_{2}$ manipulation. Neuroimage 39(1):107-118

62. Friedrich MG, Karamitsos TD (2013) Oxygenation-sensitive cardiovascular magnetic resonance. J Cardiovasc Magn Reson 15(1):1-11

63. Buxton RB (2013) The physics of functional magnetic resonance imaging (fMRI). Rep Progress Phys Phys Soc (Great Britain) 76(9):096601

64. Teixeira T, Hafyane T, Stikov N, Akdeniz C, Greiser A, Friedrich MG (2016) Comparison of different cardiovascular magnetic resonance sequences for native myocardial T1 mapping at $3 \mathrm{~T}$. J Cardiovasc Magn Reson 18(1):65

65. Piechnik SK, Evans J, Bary LH, Wise RG, Jezzard P (2009) Functional changes in CSF volume estimated using measurement of water T2 relaxation. Magn Reson Med 61(3):579-586

66. Nagel E, Puntmann VO (2016) Is myocardial native T1 the one answer for all?*. JACC Cardiovasc Imaging 9(1):37-39

67. Kramer CM, Chandrashekhar Y, Narula J (2016) The tissue issue. T1 mapping and the myocardium. JACC Cardiovasc Imaging 9 (1):88-90

68. Salerno M, Sharif B, Arheden H, Kumar A, Axel L, Li D, Neubauer $S$ (2017) Recent advances in cardiovascular magnetic resonance: techniques and applications. Circ Cardiovasc Imaging. doi:10.1161/CIRCIMAGING.116.003951

69. Paiman EHM, Lamb HJ When should we use contrast material in cardiac MRI? J Magn Reson Imaging. doi:10.1002/jmri.25754

70. Dastidar AG, Bucciarelli-Ducci C (2016) Coronary artery disease imaging: What is the role of magnetic resonance imaging? Dialogues Cardiovasc Med 21:267-276 